\title{
Buckling Paradox and Anisotropic Plastic Plate Bifurcation
}

\author{
Suresh Shrivastava \\ Department of Civil Engineering and Applied Mechanics \\ McGill University \\ Montreal, Quebec, Canada H3A 2K6 \\ Email: suresh.shrivastava@mcgill.ca
}

\begin{abstract}
Anisotropic elastic/plastic plate bifurcation is investigated. The pre-bifurcation loading consists of biaxial stresses along $\xi v$ axes parallel to the sides of a simply supported rectangular plate: $\sigma_{v v}=\alpha \sigma_{\xi \xi},-1 \leq \alpha \leq 1$. The $\xi v$ axes are at an angle $\beta$ with the $x y$ principal axes of anisotropy. Analytical variational method is used together with Hill's theory of anisotropic strain-hardening plasticity. Bifurcation stresses are determined for the anisotropic, and the classical isotropic incremental and deformation theories of plasticity, for equibiaxial compression $(\alpha=1)$, equal compression and tension $(\alpha=-1)$, and uniaxial $(\alpha=0)$ cases. The plastic plate buckling paradox is examined for each of the cases.
\end{abstract}

Keywords-anisotropic plastic bifurcation, isotropic plastic bifurcation (incremental, deformation), buckling paradox

\section{INTRODUCTION}

The plate plastic buckling paradox originated from the work of Handelman and Prager [1]. They found that the bifurcation stresses predicted by the isotropic Mises incremental theory, the "correct" theory of strain hardening plasticity, were absurdly higher than those from the "incorrect" deformation theory of plasticity. Experiments generally favour the predictions of the deformation theory. Hence, the plastic plate buckling paradox: a correct theory yields the wrong results, while an incorrect one gives the right results. Onat and Drucker [2] explained the paradox by showing that by taking "unavoidable" out-of-plane geometric imperfection into account in a nonlinear growth analysis of a plate, the incremental theory gives maximum loads matching the deformation theory bifurcation loads. The growth analysis is quite complicated, and can only be done numerically; efforts have been made to lower the buckling loads of the incremental theory by various means, but without success.
Here, a bifurcation analysis for plate buckling is performed by considering the anisotropic behaviour of the plate material. For this purpose Hill's incremental theory [3] for anisotropic strain hardening of sheet metals is used. The $x$ principal axis of anisotropy is taken as the rolling direction; $y$ is the transverse principal axis. These anisotropy axes are assumed to remain fixed, uninfluenced by the loading of the plate for bifurcation. Plane stress conditions are assumed to prevail. The loading is biaxial $\sigma_{v v}=\alpha \sigma_{\xi \xi},-1 \leq \alpha \leq 1$, with $\xi v$ axes parallel to the sides of the rectangular plate, simply supported at $\xi=0, a$ and at $v=0, b$. The $\xi v$ axes are at an angle $\beta$ (which may or may not be zero) with respect to the xy principal axes of anisotropy. Three cases are considered: (1) equibiaxial, (2) equal compression tension, and (3) uniaxial compression. The anisotropic bifurcation stresses are compared with those from the isotropic incremental and deformation theories of plasticity.

\section{ANISOTROPIC CONSTITUTIVE RELATIONS}

The stresses along the anisotropy axes are

$$
\begin{aligned}
\sigma_{x x} & =\sigma_{\xi \xi} \cos ^{2} \beta+\sigma_{v v} \sin ^{2} \beta \\
\sigma_{y y} & =\sigma_{\xi \xi} \sin ^{2} \beta+\sigma_{v v} \cos ^{2} \beta \\
\sigma_{x y} & =\left(\sigma_{\xi \xi 1}-\sigma_{v v}\right) \cos \beta \sin \beta \\
\sigma_{y x} & =\left(\sigma_{\xi \xi}-\sigma_{v v}\right) \cos \beta \sin \beta
\end{aligned}
$$

The anisotropic yield criterion of Hill [3] is

$$
2 f\left(\sigma_{i j}\right)=(G+H) \sigma_{x x}^{2}+(F+H) \sigma_{y y}^{2}-2 H \sigma_{x x} \sigma_{y y}+2 N \sigma_{x y}^{2}=1
$$


where

$G+H=\frac{1}{X^{2}}, F+H=\frac{1}{Y^{2}}, 2 H=\frac{1}{X^{2}}+\frac{1}{Y^{2}}-\frac{1}{Z^{2}}, 2 N=\frac{1}{T^{2}}$.

$X, Y, Z$ and $T$ are the current direct and shear yield stresses.

For isotropy $Z=Y=\sqrt{3} T=X$.

Normality rule gives the plastic strain increments: $d \varepsilon_{i j}^{P}=d \lambda \frac{\partial f}{\partial \sigma_{i j}}$ where $d \lambda=d W^{P}=\sigma_{i j} d \varepsilon_{i j}^{P}$. Explicitly:

$d \varepsilon_{x x}^{P}=d W^{P}\left\{\left(G \sigma_{x x}+H\left(\sigma_{x x}-\sigma_{y y}\right)\right\}\right.$,

$d \varepsilon_{y y}^{P}=d W^{P}\left\{\left(F \sigma_{y y}-H\left(\sigma_{x x}-\sigma_{y y}\right)\right\}\right.$,

$d \varepsilon_{z z}^{P}=-d W^{P}\left(G \sigma_{x x}+F \sigma_{y y}\right)$

$d \varepsilon_{x y}^{P}=2 d W^{P} N \sigma_{x y}, d \varepsilon_{y z}^{P}=0, d \varepsilon_{z x}^{P}=0$

The elastic increments, assumed isotropic, are

$$
\begin{aligned}
d \varepsilon_{i j}^{E} & =\frac{1+v}{E} d \sigma_{i j}-\frac{v}{E} d \sigma_{k k} \delta_{i j}, d \varepsilon_{i i}^{E}=\frac{1-2 v}{E} d \sigma_{k k} \\
d \sigma_{i j} & =\frac{E}{1+v}\left\{d \varepsilon_{i j}^{E}+\frac{v}{(1-2 v)} d \varepsilon_{k k}^{E} \delta_{i j}\right\}
\end{aligned}
$$

The total strain increments, elastic plus plastic, are

$d \varepsilon_{i j}=\frac{1+v}{E} d \sigma_{i j}-\frac{v}{E} d \sigma_{k k} \delta_{i j}+d W^{P} \frac{\partial f}{\partial \sigma_{i j}}$

Now, as defined by Hill, the equivalent stress and the equivalent plastic strain increments are:

$\sigma_{e q}=\sqrt{\frac{3}{2} \frac{h^{2}}{\left(F_{0}+G_{0}+H_{0}\right)}}, d \varepsilon_{e q}^{P}=\sqrt{\frac{2}{3} \frac{\left(F_{0}+G_{0}+H_{0}\right)}{h^{2}}} d W^{P}$

where $F_{0}, G_{0}, H_{0}$ and $N_{0}$ are related to the initial yield stresses in direct and shear stresses, and

$$
h=\sqrt{\left(F_{0}+H_{0}\right) \sigma_{y y}^{2}+\left(G_{0}+H_{0}\right) \sigma_{x x}^{2}-2 H_{0} \sigma_{x x} \sigma_{y y}+2 N_{0} \sigma_{x y}^{2}} \geq 1
$$

is the non-dimensional hardening function, assumed common for all yield stresses:

$$
\begin{aligned}
& X=h X_{0}, Y=h Y_{0}, Z=h Z_{0}, T=h T_{0} \text { or, } \\
& F_{0}=h^{2} F, G_{0}=h^{2} G, H_{0}=h^{2} H, N_{0}=h^{2} N .
\end{aligned}
$$

Also

$d W^{P}=\sigma_{m n} d \varepsilon_{m n}^{P}=\sigma_{e q} d \sigma_{e q} \frac{d \varepsilon_{e q}^{P}}{d \sigma_{e q}}=\frac{d\left(\sigma_{e q}^{2}\right)}{2} \frac{(\lambda-1)}{E}$

where $\lambda=E / E_{t}, E$ and $E_{t}$ are the elastic and tangent moduli from a uniaxial stress-strain curve of the material. Hence

$$
d \varepsilon_{i j}=\frac{(1+v) d \sigma_{i j}-v d \sigma_{k k} \delta_{i j}}{E}+\frac{3(\lambda-1) d h^{2}}{8 E h^{2}\left(F_{0}+G_{0}+H_{0}\right)} \frac{\partial h^{2}}{\partial \sigma_{i j}}
$$

where $\frac{\partial f}{\partial \sigma_{i j}}=\frac{1}{2 h^{2}} \frac{\partial h^{2}}{\partial \sigma_{i j}}$ has been substituted.

For plane stress behaviour $d \sigma_{z z}=0$, one has:

$d \varepsilon_{z z}=-\frac{v}{E}\left(d \sigma_{x x}+d \sigma_{y y}\right)-\left(d \varepsilon_{x x}^{P}+d \varepsilon_{y y}^{P}\right)$.

The above relations can be inverted to express $d \sigma_{i j}$ in terms of $d \varepsilon_{i j}$. The results can be expressed as:

$$
\left\{\begin{array}{l}
d \sigma_{x x} \\
d \sigma_{y y} \\
d \sigma_{x y} \\
d \sigma_{y x}
\end{array}\right\}=\left[\begin{array}{llll}
C_{11} & C_{12} & C_{13} & C_{14} \\
C_{21} & C_{22} & C_{23} & C_{24} \\
C_{31} & C_{32} & C_{33} & C_{34} \\
C_{41} & C_{42} & C_{43} & C_{44}
\end{array}\right]\left\{\begin{array}{l}
d \sigma_{x x} \\
d \sigma_{y y} \\
d \sigma_{x y} \\
d \sigma_{y x}
\end{array}\right\}, C_{j i}=C_{i j}
$$

The incremental moduli $C_{i j}$ depend on the stresses at bifurcation, $\lambda=E / E_{t}$, and the anisotropic parameters $F_{0}, G_{0}, H_{0}, N_{0}$. Referred to the $\xi v$ axes:

$$
\left\{\begin{array}{l}
d \sigma_{\xi \xi} \\
d \sigma_{v v} \\
d \sigma_{\xi v} \\
d \sigma_{v \xi}
\end{array}\right\}=[R]\left\{\begin{array}{l}
d \sigma_{x x} \\
d \sigma_{y y} \\
d \sigma_{x y} \\
d \sigma_{y x}
\end{array}\right\},[R]=[R(\beta)],[R]^{-1}=[R]^{T}=[R(-\beta)],
$$

$[R]=\left[\begin{array}{cccc}\cos ^{2} \beta & \sin ^{2} \beta & \cos \beta \sin \beta & \cos \beta \sin \beta \\ \sin ^{2} \beta & \cos ^{2} \beta & -\cos \beta \sin \beta & -\cos \beta \sin \beta \\ -\cos \beta \sin \beta & \cos \beta \sin \beta & \cos ^{2} \beta & \sin ^{2} \beta \\ -\cos \beta \sin \beta & \cos \beta \sin \beta & \sin ^{2} \beta & \cos ^{2} \beta\end{array}\right]$

where, as defined before, $\beta$ is the angle between the $\xi$ axis of loading and the $X$ principal axis of anisotropy. Thus

$$
\left\{\begin{array}{l}
d \sigma_{\xi \xi} \\
d \sigma_{v v} \\
d \sigma_{\xi v} \\
d \sigma_{v \xi}
\end{array}\right\}=\left[\begin{array}{llll}
B_{11} & B_{12} & B_{13} & B_{14} \\
B_{12} & B_{12} & B_{12} & B_{12} \\
B_{12} & B_{12} & B_{12} & B_{12} \\
B_{12} & B_{12} & B_{12} & B_{12}
\end{array}\right]\left\{\begin{array}{l}
d \varepsilon_{\xi \xi} \\
d \varepsilon_{v v} \\
d \varepsilon_{\xi_{v}} \\
d \varepsilon_{v \xi}
\end{array}\right\},[B]=[R]^{T}[C][R]
$$

$B_{j i}=B_{i j}$ are too complicated to express explicitly. 


\section{CLASSICAL ISOTROPIC THEORIES}

The incremental stress strain relations based upon the $J_{2}$ (Mises) yield criterion are given by [4]:

$\sigma_{\xi \xi}=B^{\prime} \varepsilon_{\xi \xi}+C^{\prime} \varepsilon_{v v} \quad \sigma_{v v}=C^{\prime} \varepsilon_{\xi \xi}+D^{\prime} \varepsilon_{v v}$

$\sigma_{\xi v}=2 F^{\prime} \varepsilon_{\xi v}, \sigma_{v \xi}=2 F^{\prime} \varepsilon_{v \xi}$

where

$B^{\prime}=\frac{E\{(\lambda+3+3 e+4 \lambda \alpha(\alpha-1)\}}{\Delta}$,

$D^{\prime}=\frac{E\left\{\left(\alpha^{2}(\lambda+3+3 e)-4 \lambda(\alpha-1)\right\}\right.}{\Delta}$,

$C^{\prime}=\frac{E\left\{2\left(1+\alpha^{2}\right)(\lambda-1+2 v)+\alpha(5+3 e-5 \lambda-4 v)\right\}}{\Delta}$,

$F^{\prime}=\frac{E}{(2+2 v+3 e)}$,

$\Delta=\left(1+\alpha^{2}-\alpha\right)\left\{\lambda(5+3 e-4 v)-(1-2 v)^{2}\right\}+$

$3 \alpha(1-\lambda+e)(1-2 v)$.

The parameters $\lambda=E / E_{t}, e=E / E_{s}-1 \quad$ where $E_{t}=d \sigma / d \varepsilon$ and $E_{s}=\sigma / \varepsilon$ are the tangent and secant moduli at the equivalent stress level $\sigma_{e q}^{\prime}$ which for the biaxial state of stress $\sigma^{\prime}{ }_{v v}=\alpha \sigma_{\xi \xi}^{\prime}$, is

$\sigma_{e q}^{\prime}=\sqrt{3 J_{2}}=\sqrt{\left(\sigma_{\xi \xi}^{\prime}\right)^{2}-\sigma_{\xi \xi}^{\prime} \sigma_{v V}^{\prime}+\left(\sigma_{v V}^{\prime}\right)^{2}}$

$=\sqrt{1-\alpha+\alpha^{2}} \times \sigma_{\xi \xi}^{\prime}$

Above are the current moduli of the $J_{2}$ deformation theory. The current moduli of the $J_{2}$ incremental theory follow by substituting $e=0$ in the above equations. Moduli for the elastic theory are obtained by substituting $e=0$ and $\lambda=1$. Thus, the constitutive relations incorporate the two isotropic strainhardening plasticity theories, and also the purely elastic behaviour. We write:

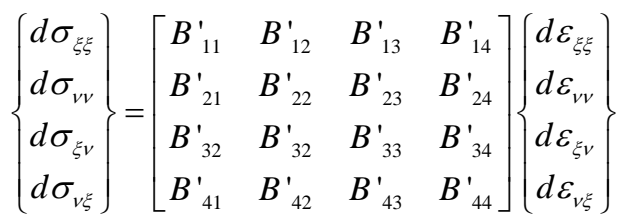

where $B^{\prime}{ }_{j i}=B^{\prime}{ }_{i j}$ are the isotropic moduli in (19) in lieu of the anisotropic moduli in (15).

\section{STRESS STRAIN CURVE}

The material is taken to be 2024S-T3 Aluminum alloy, used in aerospace, with a stress strain curve:

$\varepsilon=\frac{\sigma}{76,500}+0.002\left(\frac{\sigma}{300}\right)^{7}$

where $\sigma$ is in MPa units [5] and $E=76,500 \mathrm{MPa}$. This is taken as the required relation between $\sigma_{e q}=\sigma$ and $\varepsilon_{e q}^{P}=\int d \varepsilon_{e q}^{p}$. Yielding begins around 170 MPa. The representation is experimentally valid up to $\sigma=310 \mathrm{MPa}$. Poisson's ratio is taken as 0.33 .

For accounting anisotropic plasticity $X_{0}=170 \mathrm{MPa}$ is taken. Other yield stresses $Y_{0}, Z_{0}, T_{0}$ are given appropriate values in terms of $X_{0}$. The angle $\beta$ between the loading and anisotropy axes is taken here to be just $0^{0}$ and $22.5^{0}$.

\section{FORMULATION FOR BIFURCAION}

Let $w(\xi, v)$ be the bifurcation transverse $Z$ displacement. Invoking the Kirchhoff kinematic hypothesis for thin plates, the incremental strains (symbol $d$ omitted hereon) due to bifurcation are

$\varepsilon_{\xi \xi}=-Z W_{\xi \xi}, \varepsilon_{v v}=-Z W_{v v}, \varepsilon_{\xi v}=-Z W_{\xi v}, \varepsilon_{v \xi}=-Z W_{v \xi}$

The stress increments are therefore

$$
\begin{aligned}
& \sigma_{\xi \xi}=-z\left(B_{11} \varepsilon_{\xi \xi}+B_{12} \varepsilon_{v v}+B_{13} \varepsilon_{\xi v}+B_{14} \varepsilon_{v \xi}\right) \\
& \sigma_{v v}=-z\left(B_{21} \varepsilon_{\xi \xi}+B_{22} \varepsilon_{v v}+B_{23} \varepsilon_{\xi v}+B_{24} \varepsilon_{v \xi}\right) \\
& \sigma_{\xi v}=-z\left(B_{31} \varepsilon_{\xi \xi}+B_{32} \varepsilon_{v v}+B_{33} \varepsilon_{\xi v}+B_{34} \varepsilon_{v \xi}\right) \\
& \sigma_{v \xi}=-z\left(B_{41} \varepsilon_{\xi \xi}+B_{42} \varepsilon_{v v}+B_{43} \varepsilon_{\xi v}+B_{44} \varepsilon_{v \xi}\right)
\end{aligned}
$$

Plastic bifurcation under increasing load (Shanley's concept) requires $B_{i j}$ to be constants over the thickness. Hence

$$
\begin{aligned}
& M_{\xi \xi}=\frac{t^{3}}{12}\left(B_{11} w_{\xi \xi}+B_{12} w_{v v}+B_{13} w_{\xi v}+B_{14} w_{v \xi}\right), \\
& M_{v v}=\frac{t^{3}}{12}\left(B_{21} w_{\xi \xi}+B_{22} w_{v v}+B_{23} w_{\xi v}+B_{24} w_{v \xi}\right), \\
& M_{\xi v}=\frac{t^{3}}{12}\left(B_{31} w_{\xi \xi}+B_{32} w_{v v}+B_{33} w_{\xi v}+B_{34} w_{v \xi}\right) . \\
& M_{v \xi}=\frac{t^{3}}{12}\left(B_{41} w_{\xi \xi}+B_{42} w_{v v}+B_{43} w_{\xi v}+B_{44} w_{v \xi}\right) .
\end{aligned}
$$


Now, the principle of virtual work requires

$$
\begin{aligned}
& \int_{0}^{a} \int_{0}^{b}\left(M_{\xi \xi} \delta w_{\xi \xi}+M_{v v} \delta w_{v v}+M_{\xi v} \delta w_{\xi v}+M_{v \xi} \delta w_{v \xi}-\right. \\
& \left.P_{1} w_{\xi} \delta w_{\xi}-\alpha P_{1} w_{v} \delta w_{v}\right) d \xi d v=0
\end{aligned}
$$

where virtual work of axial loads $P_{1}$ and $P_{2}=\alpha P_{1}$ per unit length has been accounted. Substitution of $M_{\xi \xi}$ etc. renders the virtual work equation as

$$
\begin{aligned}
& \frac{t^{3}}{12} \int_{0}^{a} \int_{0}^{b}\left\{\left(B_{11} w_{\xi \xi}+B_{12} w_{v v}+B_{13} w_{\xi v}\right) \delta w_{\xi \xi}+\left(B_{21} w_{\xi \xi}+B_{22} w_{v v}+\right.\right. \\
& \left.B_{23} w_{\xi v}+B_{24} w_{v \xi}\right) \delta w_{v v}+\left(B_{31} w_{\xi \xi}+B_{32} w_{v v}+B_{33} w_{\xi v}+\right. \\
& \left.B_{34} w_{v \xi}\right) \delta w_{\xi v}+\left(B_{41} w_{\xi \xi}+B_{42} w_{v v}+B_{43} w_{\xi v}+B_{44} w_{v \xi}\right) \delta w_{v \xi} \\
& \left.-P_{1}\left(w_{\xi} \delta w_{\xi}+\alpha w_{v} \delta w_{v}\right)\right\} d \xi d v=0
\end{aligned}
$$

\section{DEERMINING THE BIFURCATION STRESS}

Galerikin procedure is used. The displacement $w$ and its variation $\delta w$ are taken of the same form, satisfying the simple support conditions around the edges. The functions

$$
\begin{aligned}
& w=\Sigma A_{i} \sin \frac{m_{i} \pi \xi}{a} \sin \frac{n_{i} \pi v}{b}, \\
& \delta w=\Sigma \delta A_{i} \sin \frac{m_{i} \pi \xi}{a} \sin \frac{n_{i} \pi v}{b}
\end{aligned}
$$

are such functions, where $m_{i}, n_{i}$ are the number of half waves in $\xi, v$ directions. The terms are such that $m_{i}+n_{i}$ is equal to $2,3,4,5,6,7,8$, giving 28 terms, a number assumed to provide sufficiently accurate values of the bifurcation stresses.

When substituted in the above virtual work equation and integrated, the arbitrary character of $\delta A_{i}$ yields a system of linear homogeneous equations of a generalized eigenvalue problem

$$
\frac{t^{3}}{12}\left[K_{1}\right]\{A\}-P_{1}\left[K_{2}\right]\{A\}=\{0\}
$$

For a chosen $\sigma_{\xi \xi}$, the problem can be cast as

$$
\left[\left[K_{1}^{\prime}\right]-\frac{b^{2}}{t^{2}}\left[K_{2}\right]\right]\{A\}=\{0\}
$$

where $\left[K_{1}^{\prime}\right]=\frac{b^{2}}{12 \sigma_{\xi \xi}}\left[K_{1}\right]$ depends on $\sigma_{\varsigma \xi}$, moduli $B_{i j}$ or $B_{i j}^{\prime}$ and $r=\frac{a}{b}$. Thus, the eigenvalue is $\frac{b^{2}}{t^{2}}$.
Mathematica programs were constructed to obtain the critical values of $\frac{t}{b}$ versus $\sigma_{\xi \xi}$ for the present incremental anisotropic theory of plasticity of Hill, and for the two classical isotropic theories.

\section{EQUIBIAXIAL COMPRESSION CASE}

The results are given for rectangular plates with $a / b=5$. The equibiaxial compressive stresses result in zero shear stress everywhere. The graphs below show gradual divergence between the incremental and deformation theory. At $t / b \approx 0.0927$ the critical stress is $310 \mathrm{MPa}$ for the incremental theory, whereas for the deformation theory it is around $286 \mathrm{MPa}$ which is lower by $7.7 \%$. This is not large difference and can be made still smaller by taking the transverse shear effects in the incremental theory. For circular SS plate [6] this difference is about $1 \%$. This means that for this equibiaxial loading the paradox is practically non-existent. For a slight anisotropy $X_{0}=Y_{0}=170 \mathrm{MPa} Z_{0}=0.9 X_{0}$ the critical stress is around $290 \mathrm{MPa}$, somewhat higher than that from the deformation theory and lower than the incremental theory. No experimental data are available for this loading.

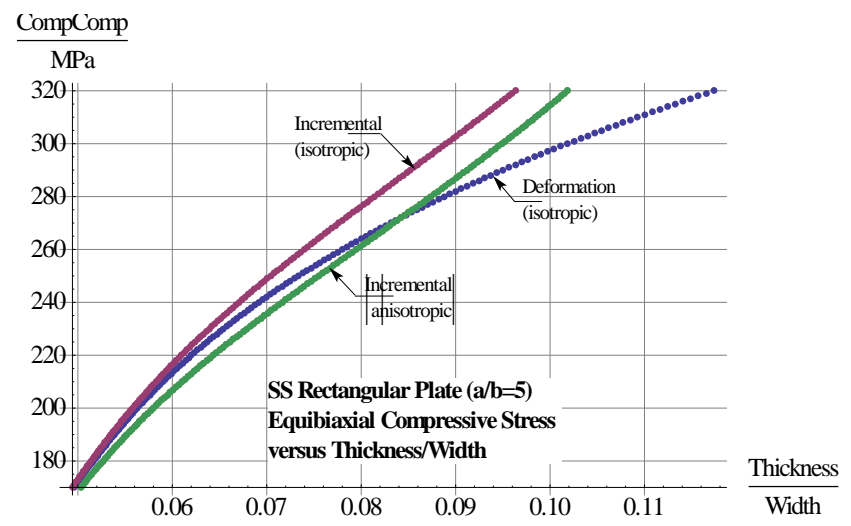

Fig 1: Critical Stress $\sigma_{\xi \xi}$ in Equibiaxial Loading

\section{EQUAL COMPRESSION AND TENSION LOADING}

Figure 2 below shows, for $a$ / $b=1$ square plates, the extreme divergence between the bifurcation stresses predicted by the isotropic incremental and and deformation theories, confirming the plastic plate buckling paradox for this case of equal compression and tension loading. The results of the incremental theory exhibit increasing stiffness with increasing $t / b$ ratio, while those of the deformation theory show markedly softening behaviour. It seems that the result of the incremental theory are too high and those of the deformation theory too low. In the absence of any experimental results for 
such loading, it is conjectured that the true values are midway between the results of the two theories.

Graphs obtained by choosing

$X_{0}=170, Y_{0}=1.3 \times X_{0}, Z_{0}=1.35 \times X_{0}, T_{0}=X_{0} / \sqrt{3} \mathrm{MPa}$

are shown for $\beta=0$ and $\beta=22.5^{\circ}$. These graphs lie between the results of the two other theories.

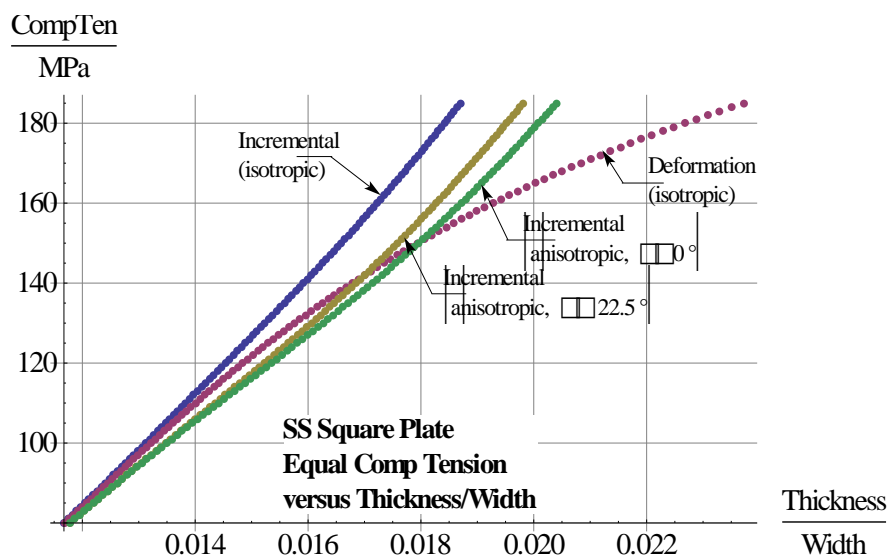

Fig. 2: Critical Stress $\sigma_{\xi \xi}$ in Equal Comp-Tension

The above anisotropic values are not unique. They were chosen by trial and error to obtain graphs midway between the graphs of the two isotropic theories, keeping in mind that the yield stresses should not be very dissimilar.

\section{UNIAXIAL COMPRESSION LOADING}

This case, for long plates, is the one most discussed, and has many experimental studies generally favoring the results of the deformation theory. It was also the case dealt by Handleman and Prager [1], and the paradox was discovered.

Figure 3 , drawn for $a / b=5$, shows the wide divergence between the results of the incremental and the deformation theories for the uniaxial loading. The anisotropic graphs are for

$X_{0}=170, Y_{0}=1.6 \times 170, Z_{0}=1 \times 170, T_{0}=1.35 \times 170 \quad \mathrm{MPa}$, and $\beta=0^{\circ}, \beta=22.5^{\circ}$.

The graphs for these anisotropic values are quite close to the critical stresses predicted by the deformation theory, especially for $\beta=0^{\circ}$.
This holds true for the entire range of stress in plastic strainhardening behaviour. For $\beta=22.5^{\circ}$ a slightly stiffer behaviour is predicted than for $\beta=0$.

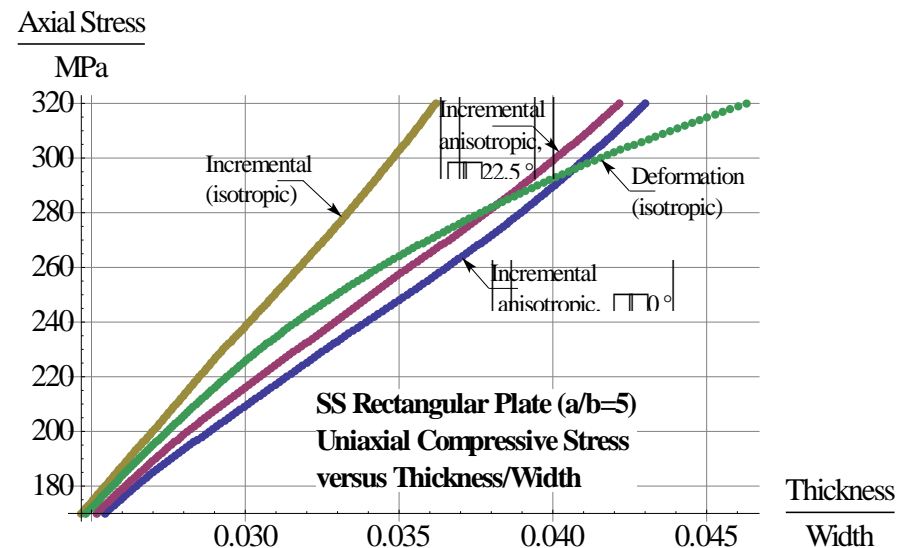

Fig. 3: Critical Stress $\sigma_{\xi \xi}$ in Uniaxial Compression

\section{CONCLUSION}

It is clear that the incremental theory, by considering the anisotropic plastic behaviour of the plate material, predicts bifurcation stresses which are close to the isotropic deformation theory results, which in turn are close to the available experimental values.

\section{REFERENCES}

[1] Handleman G. H. and Prager. W. 1949. Plastic buckling of a rectangular plate under edge thrusts: NACA Report 946.

[2] Onat E. T. and Drucker. D. C. 1953. Inelastic instabilities and incremental theories of plasticity: Journal of the Aeronautical Sciences, vol. 20, pp. 181-186.

[3] Hill R. 1950. The Mathematical Theory of Plasticity, Oxford University Press, London.

[4] Shrivastava S. and Mayenba B. M. 2013. Plastic Buckling Paradox in Biaxial loading. Proceedings of the $25^{\text {th }}$ Cancam, Western University, London, Ontario.

[5] Seide P. and Stowell E. Z. 1948. Elastic and plastic buckling of simply supported solid-core sandwich plates in compression: NACA Technical Report, NACA 967.

[6] Bahous A. 2014. Plastic buckling of circular plates on a partial elastic foundation: M. Eng. Thesis, McGill University. 